death. Of the lectures Koenigsberger says: “(Sie) bilden jetzt die ausgezeichnetsten Lehrbücher einzelner Theile der mathematischen Physik." Nothing could be truer. And may we not in all seriousness ask the question whether these live works of a master genius may not be more inspiring and really better text-books for the student than the work of some lesser person, no matter how careful and industrious he be? Of the biography let us add that it ends with consummate inspiration in the words which Helmholtz himself applied to Goethe and Beethoven : "Wir verehren in ihnen einen Genius, einen Funken göttlicher Schöpferkraft, welcher über die Grenzen unseres verständig und selbstbewusst rechnenden Denkens hinausgeht. Und doch ist der Künstler wieder ein Mensch, wie wir, in welchem dieselben Geisteskräfte wirken wie in uns selbst, nur in ihrer eigenthümlichen Richtung reiner, geklärter, in ungestörterem Gleichgewichte, und indem wir selbst mehr oder weniger schnell und vollkommen die Sprache des Künstlers verstehen, fühlen wir, dass wir selbst Teil haben an diesen Kräften, die so Wunderbares hervorbrachten."

Edwin Bidwell Wilson.

Yale University, New Haven, Conn.,

October 8, 1906.

\title{
PIERPONT'S THEORY OF FUNCTIONS.
}

The Theory of Functions of Real Variables, Volume I. By James Pierpont, Professor of Mathematics in Yale University. Boston, Ginn and Company, 1905 . xii $+560 \mathrm{pp}$.

Since the time of Weierstrass the so-called rigorous style in mathematical writing has increased constantly in favor until in recent years it has become a commonplace instead of a rarity. Such myriads of microscopic $\epsilon$ 's and $\delta$ 's have penetrated our mathematical thinking that it would be impossible to rid the system of them entirely even if it were desirable to do so. A return to the externals of the intuitive style of writing would, however, enable any one individual to obtain a conception of a much wider range of investigations, and it seems possible that such a return may be made after mathematicians have come to an understanding acknowledged by all of the precise meaning 
of the various notions of continuity and limits used in analysis. Many of the details now necessary in a rigorous exposition could then be omitted. Before this can happen, however, books of the general character of the one with which this review is concerned must become familiar reading.

Professor Pierpont's book is the first of two volumes in which he will present the subject matter of his lectures at Yale University on advanced calculus and the theory of functions of real variables. The contents may be roughly divided into three parts, the first on the real number system and the real exponential and logarithmic functions, the second on differential calculus with chapters on point aggregates and implicit functions, and the third on integration, including a more than usually complete discussion of improper integrals and integrals involving a parameter. The purpose which the author had in mind is made clear in one of the paragraphs of the preface. After a statement that the American student about to enter into graduate work often understands much of the formal processes of the calculus without having a clear knowledge of the conditions under which their applications are justifiable, he writes :

"The problem therefore arises to examine carefully the conditions under which the theorems and processes of the calculus are correct and to extend as far as possible or useful the limits of their applicability."

Some of the chapters deserve especial mention on account of their clearness and finish. For example, it would be hard to find more attractive accounts of the theory of implicit functions, indeterminate forms, and the definition and properties of a definite integral than are given in Chapters IX, X, and XII, respectively. On the other hand other parts of the book impress one as being relatively unfinished and somewhat overcomplicated. Among these are the sections devoted to integrals involving a parameter, which will be mentioned later. The pedagogical principle that clearness is attained by proving special cases of theorems first, instead of treating them as corollaries to the general case, has caused the book to be larger than it would otherwise have been, and it is doubtful if after all the advantage gained is very great.

Among the most interesting features of the book are the sections entitled "Criticism." As the author states in the preface, nothing stimulates the student's critical sense more powerfully than asking him to detect the flaws in a piece of reasoning 
which at an earlier stage of his training he considered correct. It is especially interesting, therefore, to find after each important discussion, sections in which the inaccuracies of the more elementary treatises are exposed. Mention may also be made here of the illustrative examples and the special theorems on limits. The former are numerous and well chosen, often being constructed to show how a theorem may fail to be true when its initial hypotheses are not satisfied. The latter occur especially in Chapters II, III, and VI, where many useful inequalities and limits are calculated.

In Chapters I and II Professor Pierpont has developed the theory of the real number system in a way which can not fail to be of interest. Supposing the theory of the system $I$ of positive integers to be known, he introduces in succession three new systems, $F, R, \Re$, the first consisting of elements $\left(a, a^{\prime}\right)$ with the properties of positive rational fractions, the second of elements $\left\{a, a^{\prime}\right\}$ with the properties of the differences $a-a^{\prime}$ of positive rational numbers, the third being the system of all real numbers. It is seen that the system $I$ is closed only under addition and multiplication, while $F$ is closed under addition, multiplication and division. $R$ and $\Re$ are closed under all the rational operations and $\Re$ has the additional property of admitting limiting processes. Each new system consists of entirely new elements but in each there is a subsystem with elements and properties in correspondence with those of the one just preceding.

In Chapter II, the irrational number is defined after Cantor to be * a sequence of rational numbers

$$
\left(a_{1}, a_{2}, a_{3}, \cdots, a_{n}, \cdots\right)
$$

having the property that for any $\epsilon>0$ there exists a positive integer $m$ such that $\left|a_{n}-a_{\nu}\right|<\epsilon$ whenever $n$ and $\nu$ are greater than $m$, or in Professor Pierpont's concise and satisfactory notation, a sequence for which

$$
\epsilon>0, m, \quad\left|a_{n}-a_{\nu}\right|<\epsilon, \quad n, \nu>m .
$$

The totality of such sequences is the real number system $\Re$.

* Professor Pierpont writes on p. 36, "every regular sequence defines a number." The difference between saying that the sequence is a number and saying that it defines a number, is slight. The former is perhaps less mysterious. 
The sections ( $\$ 112-123)$ in which it is pointed out that the numbers of $\Re$ and the points of a line are in one-to-one correspondence are not very convincing because to make a logical demonstration of this fact it would be necessary to state explicitly the postulates which define a geometric continuum. As far as the applications in other parts of the book are concerned it would have sufficed to show that the points of a line can be used as a kind of notation for the real number system. Indeed much of graphic mathematics which seems intuitive would be precise if the graphs were regarded merely as notations for ideas elsewhere sharply defined. In the latter part of the chapter the definition of a Dedekind partition of the rational numbers is defined and the correspondence of these to the numbers of $\Re$ is shown.

Taken altogether, Professor Pierpont's treatment of the number system is a very suggestive one, especially valuable from the pedagogic standpoint. There are a few places where improvement seems possible. On page 24 one might infer that the properties $1^{\circ}, 2^{\circ}$ characterize a sequence. That this is not true is shown by the example*

$$
a_{1}, a_{2}, a_{3}, \cdots, a_{n}, \cdots ; b_{1}, b_{2}, \ldots, b_{n}, \ldots,
$$

which has the properties referred to. These properties, however, have but slight importance in the following pages, as the notion of sequence actually used has always been a set of elements in one-to-one correspondence with the positive integers. In connection with the definitions of order and the algebraic operations on irrational numbers some essential points have been omitted. It should be shown (page 39) that any two numbers $\alpha, \beta$ satisfy one and but one of the relations $\alpha \equiv \beta$. Inasmuch as the same number has an infinity of different representations, the discussion is hardly complete without a proof that the order relations and the result of any algebraic operation are independent of the particular representations chosen for the numbers involved. Lack of space would render it inadvisable to give a complete treatment of the number system from the standpoint of the theory of assemblages, but in the sections in which the properties of $R$ and $\Re$ are discussed it would have been easy to point out especially those which as postulates characterize these systems categorically.

* Huntington, "Types of serial order," Annals of Math., 2d series, vol. 6, p. 169. 
In Chapter III the theory of the exponential $a^{z}$ is based upon the equation $x^{n}=a, n$ a positive integer. This equation determines uniquely a Dedekind partition which defines a unique position root $\rho$, designated $a^{1 / n}$. The definition of $a^{z}$ when $z$ is any rational number follows immediately, and when $z$ is an irrational number $\left(r_{1}, r_{2}, \cdots, r_{n}, \cdots\right), \alpha^{z}$ is the number $\left(a^{r_{1}}, a^{r_{2}}, \cdots, a^{r_{n}}, \cdots\right)$. Similarly the theory of the logarithm is based upon the equation $b^{x}=a$.

With Chapter IV begins the part of the book devoted to the theory of functions. Like Chapter $\mathrm{V}$ it is largely descriptive in character, the one being devoted to the explanation of the fundamental notions and notations concerning functions, and the other serving the same purpose for point aggregates. Attention need only be called to an interesting but much abbreviated proof that the trigonometric functions are not algebraic, to a very useful distinction between never decreasing (increasing) or monotone functions and always increasing (decreasing) or univariant functions, and to the notations $D(a), V(a)$ introduced on page 153. By $D_{\rho}(a)$ is meant the neighborhood $a-\rho \leqq x \leqq a+\rho$ of the point $a$, and when only the points of a particular aggregate $\mathfrak{A}$ are under consideration, $V(a)$ stands for the points of $\mathfrak{A}$ which are in $D(a)$. The symbols $D^{*}(a)$ and $V^{*}(a)$ have the same meanings except that in each case the point $a$ itself is excluded. The definition of the limit of a function may now be stated : the function $f(x)$ defined over an aggregate $\mathfrak{A}$ is said to have a limit $\eta$ as $x$ approaches $a$, if for any $D(\eta)$ there exists a $V^{*}(a)$ with the property that whenever $x$ is in $V^{*}(a)$ the value of $f(x)$ is in $D(\eta) . \quad$ It is evident that this definition holds also when $\eta$ or $a$ is infinite, provided that proper meanings are assigned to $D(+\infty)$ and $V(+\infty)$. One needs only to notice the places in Professor Pierpont's book where many special cases of theorems on limits have been or might have been collected in a single statement and proved with a single proof by means of these notations, in order to understand how really serviceable they may be. At the end of the chapter, Cantor's example of an aggregate which is perfect in an interval $\mathfrak{A}$ but does not contain all the points of $\mathfrak{A}$, is given.

So far no mention has been made in the book of limits of functions. The fundamental definition given first in Chapter VI is what may be called the sequence definition. A function $f(x)$ is said to have the limit $\eta$ as $x$ approaches $a$ if the values 
$f\left(a_{n}\right)(n=1,2, \cdots)$ have the limit $\eta$ for every sequence $a_{n}$ such that $a_{n} \neq a$ and $\lim a_{n}=a$. This definition has the advantage that it can be applied when $\eta$ or $\alpha$ is infinite, and the other well-known criteria for the existence of a limit given in the succeeding sections are readily derived from it. It is to be regretted that the author has not made more use of the notations $D(a), \quad V(a)$ described above. Much space would have been saved here $(\S 280-283)$ and in later proofs, as for example that of $\S 324$, by grouping all the special cases involving infinites into a general one. The sections explaining the geometric interpretations of the criteria for limits are very helpful. The discussion of iterated limits is brief, only one criterion being given, although the later chapters contain many instances of iterated limits of various kinds for which special criteria have been derived in each case. Perhaps one reason why these have not all been treated by similar methods is that the criterion given by Professor Pierpont demands the existence of the double limit

$$
\mathbf{L}_{\substack{x=a \\ y=b}} f(x, y)
$$

which for example in the theorem

$$
\int_{a}^{b} \frac{\partial f(x y)}{\partial y} d x=\frac{\partial}{\partial y} \int_{a}^{b} f(x y) d x
$$

is not of primary interest. In this connection attention may well be called to a theorem of Professor E. H. Moore* to the effect that the two iterated limits

$$
\mathbf{L}_{y=b} \mathbf{L}_{x=a} f(x, y) \text { and } \mathbf{L}_{x=a} \mathbf{L}_{y=b} f(x, y)
$$

exist and are equal, provided that the two inner limits exist, and that one of them, say $\mathbf{L}_{x=a} f(x, y)$, is approached uniformlyin the neighborhood of $y=b$.

On pages 202-205, after a section on uniform convergence, some curious and interesting analytical representations of special functions are given. For example, the function of $x$ whose

* Lectures on advanced integral calculus, The University of Chicago, autumn quarter, 1900 ; unpublished. The theorem was also stated at the Colloquium of the American Mathematical Society, held in New Haven, Conn., Sept. 5-8, 1906. 
value is +1 for $x>0,-1$ for $x<0$, and 0 for $x=0$, denoted by signum $x$, is represented in the form

$$
\operatorname{sgn} x=\frac{2}{\pi} \lim _{n=\infty} \arctan n x .
$$

The often-quoted function of Dirichlet, which is equal to $a$ for rational values of $x$, and to $b$ for irrational values, has then the representation

$$
y=a+(b-a) \lim _{n=\infty} \operatorname{sgn}\left(\sin ^{2} n ! \pi x\right) .
$$

After the notion of limit of a function has been made clear, the definition of a continuous function is an easy matter. In Chapter VII properties of a continuous function, such as the fact that it takes on all values between its upper and lower limits, are derived in a very attractive way, and examples are given illustrating various kinds of discontinuties.

The title of Chapter VIII is "Differentiation." The derivative is defined as usual in the more elementary books without the use of the right and left upper and lower derivatives of Dini, although the examples given of functions not derivable at certain points would serve well to illustrate them. The rules governing the use of derivatives and differentials are derived with especial care and the weaknesses common in the calculus text-books are criticised. In the conclusion of the theorem on Taylor's formula (page 255), the word " in" in the phrase "Then for any $x$ in $\mathfrak{U}$ ", should be replaced by within, according to the author's usage. For the expression on the right hand side of equation (1) is not well-defined at $x=a$ on account of the derivative $f^{(n)}(c)$ which occurs in it. In the latter part of the chapter, devoted to functions of several variables, the theorem on the reversibility of differentiation for a function $f(x, y)$ could be much improved. Under the assumption that $f_{x}^{\prime}$ exists on $y=b, f_{y}^{\prime}$ on $x=a$, and that one of them is approached uniformly, it follows as a corollary to the theorem of Moore mentioned above, that the second derivatives $f_{x y}^{\prime \prime}, f_{y x}^{\prime \prime}$ exist at $(a, b)$ and are equal. The hypotheses which Professor Pierpont makes in order to prove the theorem refer to a region about the point $(a, b)$, as must necessarily be the case if one uses the law of the mean as he does in the proof. A similar remark applies to the theorem of page 271 in which the con- 
ditions are set down for a function $f(x, y)$ to be totally differentiable, that is, to be expressible in the form

$$
f(a+h, b+k)=h f_{x}^{\prime}(a, b)+k f_{y}^{\prime}(\alpha, b)+\alpha h+\beta k,
$$

in the neighborhood of the point $(a, b)$, where $\alpha$ and $\beta$ approach zero with $h$ and $k$. The proof can in fact be made without using the law of the mean on the assumptions that on $x=a$ the derivative $f_{x}^{\prime}$ exists, is approached uniformly, and at $y=b$ is continuous, while the derivative $f_{y}^{\prime}$ exists at $(a, b)$. After the proof of Taylor's formula for functions of several variables an interesting example is given, which illustrates a case in which the expansion does not hold.

The method applied in Chapter IX to implicit functions is a method of approximation due to Goursat. It is somewhat artificial but has the advantage of demanding the existence of the derivative with respect to the dependent variable only. Usually for an equation in the form

$$
F(x, u)=0,
$$

the hypothesis is made that the function $F$ has both of its first partial derivatives continuous. Professor Pierpont's is apparently the only one of the standard books in which only the derivative with respect to $u$ is required. Systems of equations are, however, treated under the usual assumptions.

The following chapter on indeterminate forms is an exceptionally valuable one because it has to do with a subject which is badly treated in the elementary books and neglected in the more advanced treatises. The theorems are clean cut and stated with a minimum of hypothesis. In the theorem of $\S 452$ the neighborhood $R D(a)$ should evidently be $R D^{*}(a)$, and at the bottom of the page the proof might have been simplified by merely referring to the theorems on the limits of a product and sum. In one or two places several theorems could have been combined into one (e.g. $\S \S 450,451$, and $\S \S 452,453)$ with the help of the author's notation for neighborhoods, already mentioned a number of times.

Chapter XI on maxima and minima is noteworthy for the generality of the theorems for functions of a single variable, and for the pointed way in which one is warned against errors common in discussing maxima and minima of several variables. The following theorem will serve to illustrate the first point: 
"Let $f(x)$ be continuous in $D(a)$. In $D^{*}(a)$ let $f^{\prime}(x)$ be finite or infinite; and never vanish throughout any interval of it. In $R D^{*}(a)$, let $f^{\prime}(x)$ be positive when not zero ; in $L D^{*}(a)$, let $f^{\prime}(x)$ be negative when not zero. Then $f(x)$ has a minimum at a."

On pages 317 and 321, Professor Pierpont urges the reader to distinguish carefully between the terms maximum of a function at a point, and maximum of a function in an interval. It would be better to use an entirely different word, for example upper limit or upper bound, to designate the latter. The use of the word extreme for either a maximum or a minimum is a helpful simplification in many places. The theorem of $\S 476$ on extremes of functions of several variables might have been proved as briefly by a method similar to that used for functions of one variable, in which only the existence and continuity of the derivatives of the $n$th order, $i$. e., the first not vanishing identically, is assumed. The examples and criticisms of $\S \S 479-483$ bring out clearly the necessity for care in the case of functions of several variables.

The last five chapters of the book are devoted to the theory of simple proper and improper integrals, and multiple proper integrals. The first of the five is introductory and contains the usual Riemann definition of a definite integral with criteria for integrability and a discussion of certain classes of functions which are integrable. A later part of the chapter is devoted to the theory of content of a linear point aggregate $\mathfrak{A}$ from a standpoint similar to that of Jordan, and to a generalization of the definition of a definite integral by means of which it is possible to integrate a function $f(x)$ over a point set $\mathfrak{B}$ as well as over an interval. The definitions of the "outer content" of Pierpont and of "l'aire extérieure" of Jordan are different, the former being the lower bound of all intervals containing points of $\mathfrak{A}$, while the latter is the lower bound of all intervals containing points of $\mathfrak{A}$ or its boundary. The generalized integral is in fact the ordinary integral, over an interval $(a, b)$ containing $\mathfrak{B}$, of an auxiliary function $g(x)$ which has the same value as $f(x)$ at points of $\mathfrak{B}$ but is elsewhere zero. The last two theorems of the chapter are stated as corollaries, but it is difficult to see that they follow easily from the ones immediately preceding.

In Chapter XIII further properties of proper integrals are developed. Among them one finds the two theorems of the mean, a proof of the fundamental theorem of integral calculus, and theorems on the change of variable in proper integrals. 
The proof of the fundamental theorem on page 370 is one of the gems of the book, and it is to be hoped that the criticism immediately following will be widely read. The theorem of $\S 543$ which states that the upper, and similarly the lower integrals of $f(x)$ and $f(\psi(u)) \psi^{\prime}(u)$, taken over corresponding intervals, are equal when $x=\psi(u)$ has a non-vanishing continuous derivative, is the key to the transformation theorems. From it the preceding theorem of $\S 540$ follows as a corollary, and it is evident also that the part of the hypothesis in this theorem which assumes the integrability of $f(\psi(u)) \psi^{\prime}(u)$ is unnecessary. In fact it can be shown that if the functions $f(x)$ and $f(\psi(u)) \psi^{\prime}(u)$ are both integrable, one needs only to assume $\psi^{\prime}(u) \geqq 0$ (or $\left.\leqq 0\right)$ instead of the stronger assumption $\psi^{\prime}(u) \neq 0$. The theorem of $\S 540$ applying to the case when $\psi^{\prime}(u)$ is continuous but not otherwise restricted is notable for its generality and the elegance of the proof. The properties of integrals depending upon a parameter, including continuity, derivability, and integrability with respect to the parameter, are discussed in the concluding pages of the chapter. On page 387, Professor Pierpont has defined a function $f(x, y)$ to be regular in a rectangle $a \leqq x \leqq b, a \leqq y \leqq \beta$, if for each particular value of $y$ it is a limited integrable function of $x$ in the interval $a \leqq x \leqq b$. It does not follow that $f(x, y)$ is limited in the whole rectangle, although for several of the proofs, notably those on pages 391 , 392 , such an assumption is necessary. On page 424 , where the use of the word regular is still further restricted, the fact that $f(x, y)$ is limited follows from $1^{\circ}$. It is readily understood that the theorems mentioned above, concerning integrals involving a parameter, are really theorems on the inversion of iterated limits in which one or both of the limiting processes is an integral. Inversion is permissible in these cases under precisely the same conditions as for ordinary limits. For example in the equation

$$
\lim _{y=\eta} \int_{a}^{b} f(x, y) d x=\int_{a}^{b} \lim _{y=\eta} f(x, y) d x
$$

the two outer limits exist and are equal if the two inner limits exist, and if one of them is approached uniformly. It is evident then that the hypothesis,

$$
\lim _{y=\eta} \int f(x, y) d x
$$


integrable, made in $\S 565$ is superfluous. Similar alterations could be made in $\S 567$ and $\S 569$ where the derivative and integral with respect to $y$ of

$$
\int_{a}^{b} f(x, y) d x
$$

are discussed. The inversion of integration is proved in the present chapter only for continuous functions, but in much more general form in the last chapter of the book.

The improper integrals considered in Chapters XIV and $\mathrm{XV}$ are those in which the integrand has a finite number of infinities, and those in which the interval of integration is infinite. The value of this part of the book lies chiefly in the thoroughness of the extension, to these two kinds of improper integrals, of the theorems well-known in the theory of proper integrals. Aside from the criteria for convergence, the properties developed are for the most part analogous to those given in the preceding chapter. At the end of Chapter XV applications are made to the $B$ and $\Gamma$-functions. Naturally the hypotheses which must here be made in order to prove theorems on integrals involving a parameter are more complicated than for the simple integrals, and the question whether or not simplifications like those mentioned in connection with the last chapter are possible, could be answered only after careful investigation.

The final chapter of the book is on multiple proper integrals and contains properties analogous to those given in the earlier chapters for the simple integrals. As before there is a section on point aggregates, in which the properties of $n$-dimensional aggregates useful in connection with the multiple integrals are developed. The theory of the content of an aggregate $\mathfrak{A}$ is made to depend upon the fundamental properties of multiple integrals by means of an auxiliary function $f(x)$, which is equal to 1 at all points of $\mathfrak{A}$ and vanishes elsewhere. The upper and lower integrals of $f(x)$ in a field containing $\mathfrak{A}$ are the upper and lower contents of $\mathfrak{A}$ respectively, and when they coincide $\mathfrak{A}$ is said to be measurable. In $\S 722$ it is shown that the notions of upper and lower multiple integrals may be generalized so that these terms have meaning when the field of integration is any limited point aggregate $\mathfrak{A}$. Furthermore the subdivisions of $\mathfrak{A}$ used in the definition need not be by planes parallel to the axes, but may be of the much more general character specified 
on page 521. The treatment of multiple integrals is somewhat more general than that of simple integrals in that many of the theorems relate to the upper and lower integrals without any assumption that the function involved is integrable. One of the principal problems of the chapter is the reduction of multiple integrals to iterated integrals. The two theorems of $\S 733$ on iterated integrals are very general, and include as a corollary a similar theorem of Pringsheim* applying to an integrable function. One of the conclusions may be stated to give an idea of the nature of the theorems:

$$
\int_{\mathfrak{Q}} f d \mathfrak{U} \leqq \int_{x_{i}} d \mathfrak{x}_{i} \int_{\mathfrak{P}_{i}} f d \mathfrak{F}_{i} \leqq \int_{x_{i}} d \mathfrak{x}_{i} \int_{\mathfrak{P}_{i}} f d \mathfrak{P}_{i} \leqq \int_{\mathfrak{U}}^{-} f d \mathfrak{A} .
$$

Here the dashes distinguish upper and lower integrals; $\mathfrak{x}_{i}$ is the projection of the aggregate $\mathfrak{A}$ on the $x_{i}$-axis and $\mathfrak{P}_{i}$ is the variable section of $\mathfrak{A}$ by the plane $x_{i}=$ const., depending therefore upon $x_{i}$. The final conclusion with regard to the reversibility of integration of a function $f(x, y)$ in a rectangle, including all the cases considered in previous chapters, is that it is possible when $f$ is a limited function whose discontinuities on any line parallel to one of the axes, and also as a whole, form a discrete aggregate. The chapter closes with theorems on the transformation of multiple integrals of any order. Hitherto these theorems seem to have been given in detail only for double integrals. $\dagger$

Princeton,

G. A. Buiss.

October, 1906.

* Encyklopädie der mathematischen Wissenschaften, II A 2, p. 105.

† See Pierpont, "On multiple integrals," Transactions Amer. Math. Society, vol. 6 (1905), p. 416. 